\title{
Sun-Burst Appearance of Neuroblastoma Metastasis on USC
}

\author{
Amit Mittal1, Balkaran Singh Hazer², Akshay Kuamr³, Anahat Pirzada4, Preeti Garg5 \\ ${ }^{1}$ Department of Oral Medicine and Radiology, MMCDSR, MM Deemed to be University, Mullana, Haryana, India. \\ ${ }^{2}$ Department of Radiodiagnosis, MMIMSR and MMCDSR, MM Deemed to be University, Mullana, Haryana, India. \\ ${ }^{3}$ Department of Radiodiagnosis, MMIMSR and MMCDSR, MM Deemed to be University, Mullana, Haryana, India. \\ ${ }^{4}$ Department of Dermatology, MMIMSR, MM Deemed to be University, Mullana, Haryana, India. ${ }^{5}$ Department of Oral \\ Medicine and Radiology, MMIMSR and MMCDSR, MM Deemed to be University, Mullana, Haryana, India.
}

\section{INTRODUCTION}

Periosteal reaction may be classified as benign or aggressive based on the time course of the initiating process. Benign periosteal reaction is a low-grade chronic irritation which allows time for the formation of normal or near-normal cortex. The cortex will be thick and dense and has a wavy or uniform appearance. Benign periosteal reactions can be seen in callus formation in a fracture or with slowly growing tumours.

Aggressive periosteal reaction is a rapid irritation process that does not allow the periosteum time to lay down and consolidate new bone to form normal cortex. The cortex may appear lamellated, amorphous, or sunburst-like. Aggressive periosteal reactions can not only be seen with malignant tumours, but also with more benign processes like infection, eosinophilic granuloma (Langerhans cell histiocytosis), aneurysmal bone cyst, osteoid osteoma, haemophilia, and trauma.

Sunburst appearance is a type of periosteal reaction giving the appearance of a sunburst secondary to an aggressive periostitis. The sunburst appearance occurs when the lesion grows too fast and the periosteum does not have enough time to lay down a new layer and instead the Sharpey's fibres stretch out perpendicular to the bone. It is frequently associated with osteosarcoma but can also occur with other aggressive bony lesions such as an Ewing sarcoma or osteoblastic metastases (e.g. prostate, lung or breast cancer), metastasis from neuroblastoma.

Periosteal reaction, also known as periostitis or periosteitis, is a nonspecific radiographic finding that occurs with periosteal irritation. Periosteal reactions may be broadly characterized as benign or aggressive, or more specifically broken down by pattern. We present a case of 1.5 years male presented with swelling on scalp over right fronto-temporal region for 3 to 4 weeks. On ultrasonographic evaluation the swelling gave sunburst appearance. We suspected metastasis and subsequently further investigations were performed which confirmed the lesion as Neuroblastoma metastasis. There are very few cases of malignancy where sunburst appearance is described on USG in malignant lesions.

\section{PRESENTATION OF CASE}

A 1.5-year-old male presented with a history of gradually increasing scalp swelling (Figure-1) over right fronto-temporal region for 3 to 4 weeks. There was history of trauma, without any history of convulsions, vomiting, limb weakness, abnormal bleeding or bladder/bowel disturbances. There was no other relevant history. The patient was sent to the department of radio-diagnosis for further investigation of the swelling.

\author{
Corresponding Author: \\ Dr. Preeti Garg, \\ Department of Oral Medicine \& Radiology, \\ MMIMSR and MMCDSR, \\ MM Deemed to be University, \\ Mullana, Haryana, India. \\ E-mail: amitmittalmri@gmail.com
}

DOI: $10.14260 /$ jemds/2019/842

Financial or Other Competing Interests: None.

How to Cite This Article:

Mittal A, Hazer BS, Kumar A, et al. Sunburst appearance of neuroblastoma metastasis on USC. J. Evolution Med. Dent. Sci. 2019;8(51):3890-3891, DOI: $10.14260 /$ jemds/2019/842

Submission 06-09-2019,

Peer Review 02-12-2019,

Acceptance 10-12-2019,

Published 23-12-2019. 


\section{Imaging Findings}

On USG, the scalp swelling appeared as heterogeneously hypoechoic in echotexture with destruction of underlying skull vault giving typical sun-burst appearance (Figure-2).

In the neck there were multiple large lymph nodal masses in supraclavicular and mediastinum. Enlarged lymph nodes were also seen in pelvis and $b / l$ inguinal region. In view of this possibility of metastasis was kept and abdominal ultrasound was performed for primary lesion. USG showed a large, solid, heterogeneous mass with internal vascularity and large areas of necrosis appearing as regions of low echogenicity in the retroperitoneal area involving the upper pole of the left kidney. Hence this lesion was reported as a malignant retroperitoneal mass with skull vault metastasis.

An abdominopelvic CT scan followed and showed a large heterogeneously enhancing soft-tissue adrenal mass with intratumoural calcifications in the left side abdomen (Figure-3), this left adrenal mass lesion is predominantly of low attenuation. The left kidney was displaced inferiorly. A left pleural thickening was also shown associated with a right chest wall mass with no involvement of the ribs.

FNAC of the metastatic lesion was also in favor of neuroblastoma and FNAC from the abdomen lesion was also confirmatory of Neuroblastoma.

\section{DISCUSSION}

Neuroblastoma (NB) is one of the most common solid tumours in infancy and childhood. Prenatal diagnosis of NB is possible and is normally diagnosed during the third trimester. This tumour originates from neural crest, may occur in the adrenal medulla and along the sympathetic ganglion chain from the neck to the pelvis.[1] $75 \%$ of NBs are located in the retroperitoneum, in either the adrenal medulla $(50 \%)$ or the paraspinal ganglia (25\%). Less than $5 \%$ arise in the neck or pelvis. $50-75 \%$ of cases present with an abdominal mass. This pathology could also be revealed by metastasis to skull a (as in the case of our patient), orbit, liver (Pepper's syndrome)or other sites.[1] $50-60 \%$ of patients with NB present with disseminated disease, this finding is more expected in patients over 1 to 2 years of age.[2] Metastases to bone marrow and bones have been described in disseminated disease.[3] We can observe two types of marrow disease: Diffuse type and nodular type.[3] Bone marrow metastases arise initially in the sinusoids where tumour cells adhere and extravasate to parenchyma, forming nodular lesions in the medullary cavity which sometime progress to diffuse lesions and progressively grow to erode trabecular bone and invade the bone cortex, engendering bone metastases. ${ }^{[4]}$ Skull metastasis has been found in up to $25 \%$ of patients with NB. NB is the most frequent malignant metastasis to the skull in children.[5] These calvarial lesions frequently enlarge to produce epidural deposits. Metastatic involvement of the skull has various possible radiographic findings: thickened bone, the "hair-onend" periosteal reaction, lytic defects, and sutural separation. ${ }^{[6,7]}$
A periosteal reactive pattern with rays of new bone and blood vessels perpendicular to the cortex is often considered characteristic of primary bone sarcomas; however, occasionally such a pattern is found roentgenographically in association with metastatic bone tumours. ${ }^{[8-10]}$ Metastases represent the most frequent cause of multiple skull lesions. They are usually secondary to breast, lung, prostate, kidney, and thyroid cancers in adults and to neuroblastoma or sarcomas in children. ${ }^{[1-4]}$ Nearly half of metastatic lesions are due to breast cancer, explaining the predominance of women with skull metastases. ${ }^{[3]}$ These lesions are usually diagnosed in the context of a known primary tumour. They are generally asymptomatic or may be revealed by a painful swelling.[2,5] In rare cases, they represent the initial manifestation of an unknown cancer or of a recurrence.[2,4] They can be encountered at any age, with a higher prevalence during the sixth and seventh decades of life.

Radiographically, a sunray lesion or sunburst appearance is common finding. However, such appearance on sonography has been described rarely in the literature, in osteosarcoma of the mandible.[11,12] This is important because the sunburst appearance was not seen on radiography but was seen on sonography.
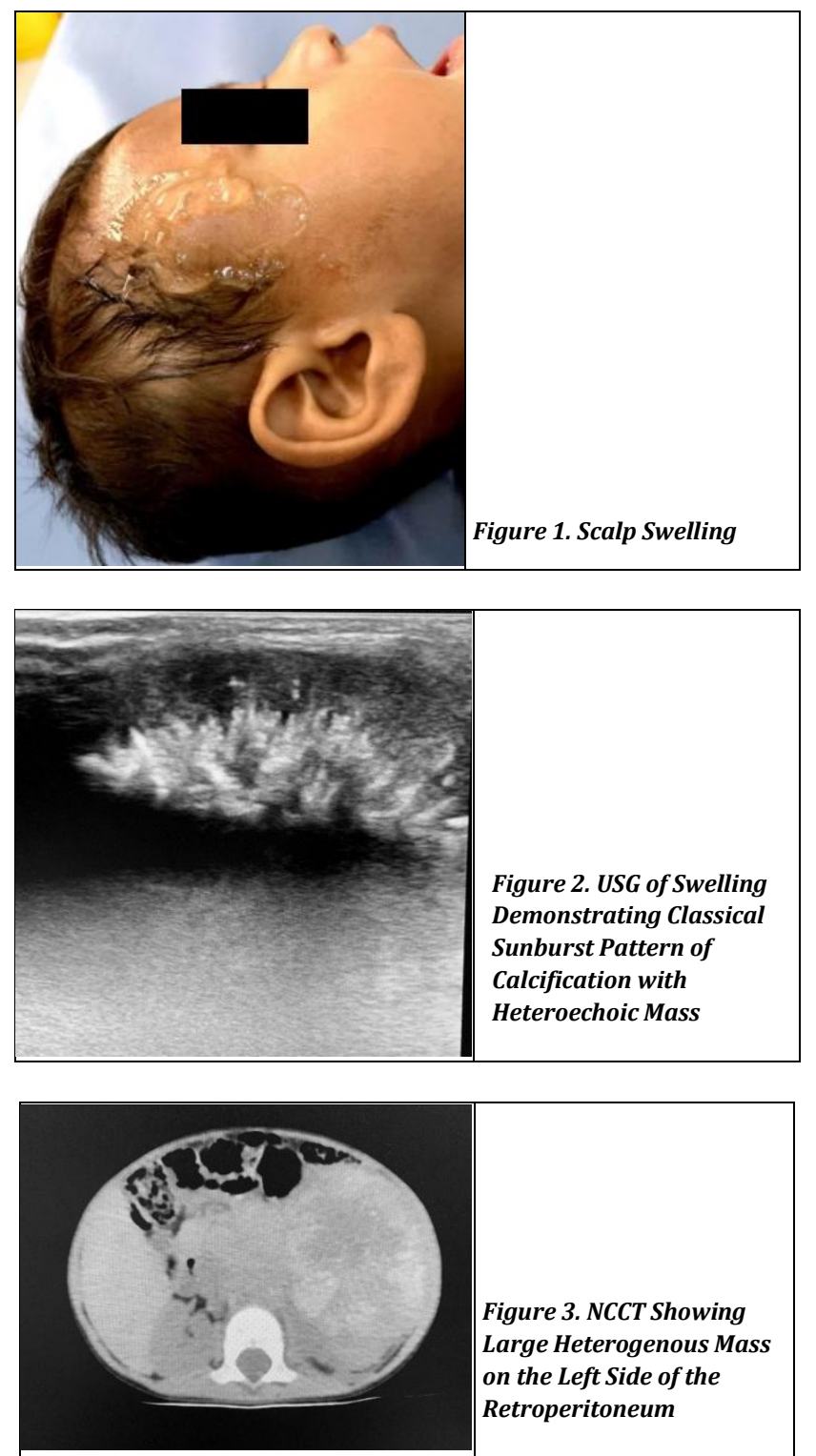

Figure 3. NCCT Showing Large Heterogenous Mass on the Left Side of the Retroperitoneum 


\section{CONCLUSIONS}

Soft tissue swellings must be first evaluated with USG and if sunburst type of periosteal reaction is seen, we should think of primary or secondary malignant pathology. Besides CT and Radiography, USG is an excellent modality to demonstrate such types of periosteal reaction.

\section{REFERENCES}

[1] Brodeur GM, Maris JM. Neuroblastoma. In: Pizzo PA, Poplack DG, eds. Principles and Practice of Pediatric Oncology. $5^{\text {th }}$ edn. Philadelphia: Lippincott Williams and Wilkins 2006.

[2] Brossard J, Bernstein ML, Lemieux B. Neuroblastoma: an enigmatic disease. Br Med Bull 1996;52(4):787-801.

[3] DuBois SG, Kalika Y, Lukens JN, et al. Metastatic sites in stage IV and IVS neuroblastoma correlate with age, tumour biology and survival. J Pediatr Hematol Oncol 1999;21(3):181-9.

[4] Tanabe M, Ohnuma N, Iwai J, et al. Bone marrow metastasis of neuroblastoma analyzed by MRI and its influence on prognosis. Med Pediatr Oncol 1995;24(5):292-9.
[5] Healy JF, Bishop J, Rosenkrantz H. Cranial computed tomography in the detection of dural, orbital and skull involvement in metastatic neuroblastoma. J Comput Tomogr 1981;5(4):319-23.

[6] Zimmerman RA, Bilaniuk LT. CT of primary and secondary craniocerebral neuroblastoma. Am J Roentgenol 1980;135(6):1239-42.

[7] Latchaw RE, L'Heureux PR, Young G, et al. Neuroblastoma presenting as central nervous system disease. Am J Neuroradiol 1982;3(6):623-30.

[8] Gaetani P, Di Ieva A, Colombo P, et al. Calvarial metastases as clinical presentation of renal cell carcinoma: report of two cases and review of the literature. Clin Neurol Neurosurg 2005;107(4):329-33.

[9] Stark AM, Eichmann T, Mehdorn HM. Skull metastases: clinical features, differential diagnosis and review of the literature. Surg Neurol 2003;60(3):219-26.

[10] Kader I, Strong M, George M. Skull destruction from intracranial metastasis arising from pulmonary squamous cell carcinoma: a case report. J Med Case Rep 2013;7:28.

[11] Ng SY, Songra A, Ali N, et al. Ultrasound features of osteosarcoma of mandible: a first report. Oral Surg Oral Med Oral Pathol Oral Radiol Endod 2001;92(5):582-6.

[12] Mittal A, Mehta V, Bagga P, et al. Sunray appearance on sonography in Ewing sarcoma of the clavicle. Journal of Ultrasound in Medicine 2010;29(3):493-5. 